\title{
Effect of Microwave Heating on Flavour Generation and Food Processing
}

\author{
G.E. Ibrahim, A.H. El-Ghorab, \\ K.F. El-Massry and F. Osman
}

Additional information is available at the end of the chapter

http://dx.doi.org/10.5772/49935

\section{Introduction}

The flavour and colour of a food product have significant impacts on consumer acceptability. Two of the challenges with microwave food products are that it is often difficult to achieve the desired flavour that matches products prepared in a conventional oven or by frying and to get the browning that the consumer expects. There are reactions that occur in those processes that do not occur when foods are heated in the microwave oven and this is part of what contributes to the lack of flavour and colour development. In trying to solve the flavour issues, it is important to understand what flavours are as well as all of the attributes of a food product that lead to consumer liking. Solving the colour problem involves an understanding of the reactions that produce colour and finding ways to get those reactions to occur. The typical browning which occurs when foods are heated by conventional means produces not only the desired brown pigments but also produces a variety of desirable flavours.

Flavours and colours generated as a result of the Maillard reaction are of critical importance for the commercial success of microwave-processed foods. Recent interest in the microwave generation of Maillard flavours and colours was a response on the part of the food industry, based on the consumer demand for fast and convenient food products. The fundamental differences between microwave and conventional heating, the composition of the food matrix, and the design of microwave ovens all seem to play a role in the inability of microwave heating to propagate colou and flavourting Maillard reactions in food products. The increased sales of microwave ovens in the last decade, especially into the North American market, provided the food industry with the impetus for renewed interest in carrying out the Maillard reaction in microwaveable food products. 


\subsection{Flavour definition}

Flavour is defined as the experience of the combined perception of compounds responsible for taste and aroma. The flavour of food is very important for its acceptability and a slight change in the odour of processed food may affect the overall quality of the product. Aromas come from low molecular weight organic compounds that can volatilize and be sensed in the nasal cavity. These compounds are not of one simple class of chemicals but rather are many different chemical types, including acids, esters, alcohols, ketones, pyrazines, thiazoles and terpenes as well as many others. The human body has a complex set of receptors that recognize both individual compounds as well as mixture of compounds to identify different flavours.

Character impact compounds are individual chemicals with a specific, recognizable aroma. Some examples are methyl anthranilate (concord grape), citral (lemon), cinnamic aldehyde (cinnamon), methyl salicylate (wintergreen) and diacetyl (butter). While these individual compounds have a characteristic odour, they do not make up the complete flavour of a product, whether naturally occurring such as in a concord grape or in a flavour added to a product. Many other compounds are also present which build the overall flavour profile. Considerable work has been done to identify the flavour compounds in different foods. There are over 170 compounds that have been identified that contribute to the flavour of a strawberry, while coffee and chocolate are much more complex with over 800 compounds identified.

\subsection{Natural versus artificial flavours}

In the United States, there is a legal definition of natural and artificial flavours.

The complete definitions are found in the Code of Federal Regulations (CFR) Title 21101.22 (Code of Federal Regulations, 2008). Artificial flavours are defined in (a)(1) as follows:

The term artificial flavour or artificial flavouring means any substance, the function of which is to impart flavour, which is not derived from a spice, fruit or fruit juice, vegetable or vegetable juice, edible yeast, herb, bark, bud, root, leaf or similar plant material, meat, fish, poultry, eggs, dairy products, or fermentation products thereof. Artificial flavour includes the substances listed in Sec. 172.515(b) and 182.60 of this chapter except where these are derived from natural sources.

Natural flavours are defined in (a)(3) of Title 21 101.22, as follows:

The term natural flavour or natural flavouring means the essential oil, oleoresin, essence or extractive, protein hydrolysate, distillate, or any product of roasting, heating or enzymolysis, which contains the flavouring constituents derived from a spice, fruit or fruit juice, vegetable or vegetable juice, edible yeast, herb, bark, bud, root, leaf or similar plant material, meat, seafood, poultry, eggs, dairy products, or fermentation products thereof, whose significant function in food is flavouring rather than nutritional. Natural flavours include the natural essence or extractives obtained from plants listed in Sec. 182.10, 182.20, 
182.40, and 182.50 and part 184 of this chapter, and the substances listed in Sec. 172.510 of this chapter.

The browning reaction will be discussed later and it is interesting to note that process flavours can be made using the browning reaction and are defined as natural since they are a product of roasting or heating.

Spices are also defined in (a)(2) of Title 21101.22 as follows:

The term spice means any aromatic vegetable substance in the whole, broken, or ground form, except for those substances which have been traditionally regarded as foods, such as onions, garlic and celery; whose significant function in food is seasoning rather than nutritional; that is true to name; and from which no portion of any volatile oil or other flavouring principle has been removed.

The regulation goes on to list a number of individual spices. It should be noted that many materials that are considered artificial are identical to those in nature: it is simply how they were produced that determines whether they are natural or artificial. As an example, diacetyl is natural if it comes from milk or is produced by fermentation (as in wine and fermented dairy products) but is artificial if it is synthesized from other chemicals. All of the chemical properties are the same no matter where the individual chemical came from. The only time that a compound can never be natural is if it has never been found from any natural source. One compound, which gives a cotton candy type flavour, is ethyl maltol. This has never been found in nature so if a flavor contains this compound, it will be at least partially artificial.

In other countries, there are different definitions as to what is natural and artificial. In some countries, there is the concept of nature identical. It states that if a compound exists in nature, then it does not matter where it comes from, it would not be considered artificial. The flavour could not be called natural but has simply been referred to as flavour. It is important that the regulations for each country be checked to understand what is allowed and how flavours added to products should be labeled.

\section{Sources of flavours}

Flavouring materials come from a variety of sources. One of the main sources is plants. The flavour materials can be present in any part of a plant including the flower, leaf, stem or bark. To be used in food products, the materials are generally extracted from the plant material to provide an isolate that is just the flavour. There are different techniques that can be used for isolation including solvent extraction (often ethanol), steam distillation and supercritical fluid extraction. Dairy products and meats and seafood can also be sources of flavouring materials. Dairy products provide a good source of base material that can be modified by enzymes to create much more concentrated flavors than are present in the natural dairy product. The enzymes break down the fats and proteins present to yield higher concentrations of the flavour compounds that represent the flavors of these dairy 
products. Some of the components of dairy flavors are short chain fatty acids including butyric acid that are unpleasant at high concentrations but help to contribute to the characteristic flavour of dairy products.

Many flavours are produced by processing, primarily with the use of heat. The subject of browning will be covered in more detail later in this chapter but will be briefly addressed here. Flavours can be created by heating one or more reducing sugars with one or more amino acids for different times and at different temperatures. Very different flavours can be produced which can be added to foods as natural flavours.

Flavours can also be produced using biotechnology. This is an area that has been explored for years to determine ways to get plants or microorganisms to produce higher quantities of flavouring materials than they do naturally. While there has been limited success by some companies to produce individual flavour compounds through this process, it has not achieved wide commercial success.

\section{Microwave versus conventional heating}

The industrial and domestic use of microwaves has increased dramatically over the past few decades. While the use of large-scale microwave processes is increasing, recent improvements in the design of high-powered microwave ovens, reduced equipment manufacturing costs and trends in electrical energy costs offer a significant potential for developing new and improved industrial microwave processes.

Microwave heating is relatively fast compared to conventional heating since it does not depend on the slower diffusion process in the latter. This property initiated the initial investigation into carrying out chemical reactions under microwave irradiation (Giguere et al., 1986). In certain cases, chemical reactions were completed in a few seconds that otherwise would have taken hours. In addition to fast rates of heating, microwaves are also more selective and components can be heated selectively in a reaction mixture compared to conventional heating. This property has been used to enhance the extraction of essential oils from plants immersed in a microwave transparent solvent (Paré et al., 1991).

Microwaves are electromagnetic waves within a frequency band of $300 \mathrm{MHz}$ to $300 \mathrm{GHz}$. In the electromagnetic spectrum (Fig. 1) they are embedded between the radio frequency range at lower frequencies and infrared and visible light at higher frequencies. Thus, microwaves belong to the non-ionising radiations.

Superheating of solvents is another phenomena that accompanies microwave heating and helps accelerate chemical reactions. Superheating refers to the increase in temperature of liquids above their boiling points while they remain completely in the liquid phase. For example, water boils under microwave heating at $105^{\circ} \mathrm{C}$ and acetonitrile (B.P. $82^{\circ} \mathrm{C}$ ) at $120^{\circ} \mathrm{C}$. A chemical reaction carried out in an open vessel in acetonitrile under microwave irradiation will be accelerated by 14 times relative to conventional heating, assuming the reaction rate doubles for every $10^{\circ} \mathrm{C}$ rise in temperature (Peterson, 1993). When chemical reactions are carried out in closed containers under microwave irradiation, the maximum 
temperature attainable is not limited to the temperature of the heating medium, as in conventional heating, but depends only on the microwave power applied and the rate at which the sample can lose heat. The extreme high temperatures attained in a closed container during microwave heating can generate extreme high pressures (especially if the reaction produces gaseous products), which can alter equilibrium product distribution according to Le Chatelier's principle (Peterson, 1993).

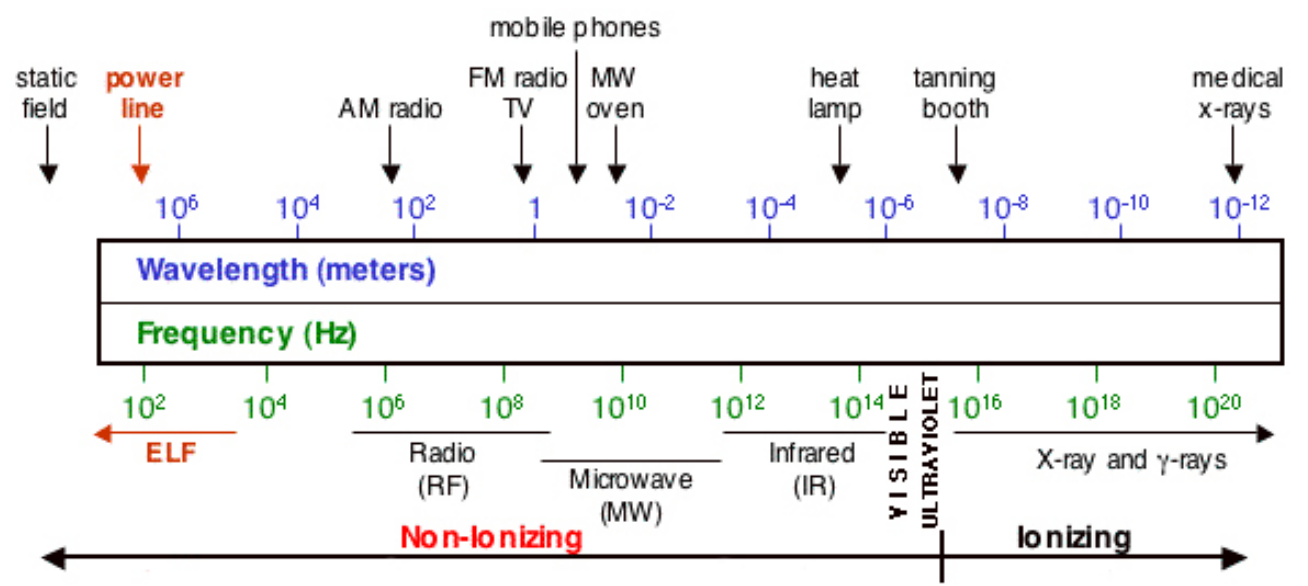

Figure 1. Electromagnetic spectrum. Additionally, the two most commonly used microwave frequency bands (at $915 \mathrm{MHz}$ and $2450 \mathrm{MHz}$ ) are sketched.

There are several major factors that impact the flavour quality of microwave food products. They primarily stem from the fact that in a conventional oven, the product is surrounded by hot air which heats the product from the outside and also dries the surface. In microwave heating, the entire product is heated at the same time but the heating may not be uniform (van Eijk, 1994). In drying the surface, it helps to reduce the rate at which volatile flavour molecules can move from inside the product to the surface and evaporate. It in a sense forms a crust that is more difficult for the flavour molecules to move through. In microwave heated products, the surface stays moist and cooler, which readily allow flavour compounds to be carried out of the food as steam is lost.

The surface of the product will also get to a higher temperature in a conventional oven. This enhances the rate of the browning reaction on the surface as this reaction goes more rapidly under lower moisture and higher temperature conditions. The browning reaction provides not only the desirable brown colour but also produces a large number of flavour compounds. In conventionally heated products, the added flavour is retained better and a large number of flavours are produced on the browned surface of the product. In products where browning is not expected, this is not an issue. If a product is simply to be reheated, the microwave does an excellent job as you are not relying on it to produce flavour. One additional factor that influences flavour development in products heated in the microwave is that they are in the oven for a much shorter period of time than those cooked in a 
conventional oven. The browning reaction takes time to develop and the product is not heated long enough for this reaction to proceed to the point where brown pigments and flavour compounds are produced. It should be noted that there are a wide variety of products where the time and temperature of heating do not create an issue for flavour development. High moisture products that are going to be reheated work very well. While some flavour will be lost during the heating process, it does not vary significantly from conventional reheating. Vegetables, with their own inherent flavor, can easily be steamed in the microwave oven.

The sensory properties of vacuum-microwave-dried and air-dried carrot slices, which were water blanched initially. The vacuum-microwave-dried carrot slices received the higher ratings for texture, odour and overall acceptability as compared to the air-dried carrot slices.

The retention of volatile components responsible for flavour was more in hot air microwave drying compared to conventional hot air drying alone. The flavour strength of garlic dried by hot air alone is $3.27 \mathrm{mg} / \mathrm{g}$ dry matter whereas the flavour strength of the garlic dried by microwave drying is $4.06 \mathrm{mg} / \mathrm{g}$ dry matter. Effect of microwave drying on the shelf life and sensory attributes (appearance, colour, odour and overall quality) of coriander (Coriander sativum), mint (Mentha spicata), fenugreek (Trigonella foenum-graceum), amaranth (Amaranthus sp.) and shepu (Peucedanum graveolens).

Amaranth had similar scores for fresh and dried ones; however, there was significant decrease for the sensory attributes of other greens. They concluded that microwave drying was highly suitable for amaranth, moderately suitable for shepu and fenugreek and less suitable for coriander and mint. Wheat samples were evaluated and the sensory characteristics of grain were assessed by the panel of 10 members. The sample produced a burnt or roasted odour when exposed for a long exposure time (180 s) but there was no significant difference in the grain odour when long exposure times were avoided.

Due to high temperature and long drying time, volatile compounds are vapourised and are lost with water vapour, resulting in significant loss of characteristic flavour in dried products. Case-hardening is a common problem in dried fruits due to rapid drying. As drying proceeds, the rate of water evaporation is faster than the rate of water movement to the product surface, hence making the outer skin dry

At air dryer temperatures, volatile flavour compounds are lost, structural changes such as case hardening may inhibit later rehydration, and extended drying times allow chemical and enzymatic reactions to degrade vitamins, flavour and colour compounds microwave dried frozen berries had a higher rehydration ratio. Microwave (MW) drying generated three unique flavou compounds (2-butanone, 2-methyl butanal, and 3-methyl butanal) while freeze-dried berries lost several, including the typical blueberry aroma, 1,8-cineole. Compared with hot-air dried berries, MW-dried cranberries have better colour, softer texture and similar

The advantages of MW blanching (MB) over conventional heat blanching methods (water or steam) include in-depth heating without a temperature gradient, and rapid inactivation of 
enzyme complexes that cause quality degradation coupled with minimal leaching of vitamins, flavours, pigments, carbohydrates, and other water-soluble components. No differences existed for flavour of green beans and mustard greens due to blanching method. In beans and mustard greens, steam blanching produced a texture equal to $\mathrm{MB}$ vegetables but chlorophyll degradation was greater. Cooking time of chicken breasts increased with decreasing power level, but cooking losses were not affected. Both sensory and instrumental tenderness (Instron compression) were best at $60 \%$ power level, while juiciness, mealiness and flavour were unaffected by power level. Convectional MW-cooked chicken was more tender, juicy and acceptable than MW-cooked chicken, avour intensity was similar. Thiamin retention ranged from $77 \%$ in conventionally cooked chicken breasts to $98 \%$ in MW-cooked chicken legs.

\section{Microwave food process design}

In designing microwave food processes and packaging, various factors that affect microwave heating of foods should be taken into consideration if the effect of uneven heating associated with the use of microwaves is to be kept under control. These factors fall into two broad categories. The first one is thermo-physical properties of the food. The second is factors associated with the dielectric characteristics of food and the field intensity distributions provided by various microwave energy applicators and heating systems.

\subsection{Physical factors}

The thermo-physical factors that require serious consideration in the design of microwave food processes and packaging systems are:

\subsubsection{Size and shape of food}

The physical size and shape of foods affect the temperature distribution within the food. This results from the fact that the intensity of the wave decreases with depth as it penetrates the food. If the physical dimensions of the food are greater than twice the penetration depth of the wave, portions of the food nearer the surface can have very high temperatures while the mid-portions are still cold. On the other hand, if the dimensions of the food are much lower than the penetration depth of the wave, the center temperature can be far higher than the temperature at the surface. This situation normally results in "the focusing effect," which results from the combined intensity of the wave (in three space dimensions) being higher at the inner portions than the outer portions of the product.

Some shapes reflect more microwaves than others. In addition, some shapes prevent increasing amounts of the waves from leaving the material by reflecting them back into the interior. For most spherical and cylindrical foods, wave focusing occurs for product diameters between 20 and $60 \mathrm{~mm}$. In rectangular foods, focusing causes the overheating of corners. Thus in package design, sharp corners are avoided and tube-shaped pans have been suggested (Giese 1992). Moreover, in foods with corners, packages are designed using 
metals or aluminum foils to reflect microwave energy away from corners and thus selectively heat some portions more.

\subsubsection{Surface area}

In microwave heating, the product temperature rises above its ambient temperature due to volumetric heating. Higher product surface area therefore results in higher surface heat loss rate and more rapid surface cooling. During microwave heating, the highest temperature is not at the surface of the product (despite the higher intensity of power absorbed there) but somewhere in the interior.

\subsubsection{Specific heat}

How much a food product will heat given a specific amount of energy depends on its heat capacity. The implication of this for microwave heating is that different food products heated together have different temperature histories. To control this, some microwave food packages are sealed tight to allow heat transfer between hotter and colder foods, thus giving similar temperature history for different foods in the same package.

\section{Microwave heating and the dielectric properties of foods}

Microwave energy is transported as an electromagnetic wave in certain frequency bands in the range between about $0.3 \mathrm{GHz}$ and $300 \mathrm{GHz}$. When microwaves impinge on a dielectric material, part of the energy is transmitted, part reflected and part absorbed by the material where it is dissipated as heat. Heating is due to 'molecular friction' of permanent dipoles within the material as they try to reorient themselves with the oscillating (electrical) field of the incident wave. The power generated in a material is proportional to the frequency of the source, the dielectric loss of the material, and the square of the field strength within it. A material is subjected to microwave energy in a device known as an applicator or cavity. Considering all these features, it is possible to identify those candidate materials and processes that can use microwave heating effectively and understand microwave ingredient interaction mechanisms. Only after such a step is taken can microwave heating be exploited fully in terms of its unique characteristics, which include the facts that no contact is required between the energy source and the target and that heating is volumetric, rapid and highly specific in nature.

International convention dictates that microwave ovens (and other industrial, scientific and medical microwave applications) operate at specific frequencies, the most favoured being 2.45 $\mathrm{GHz}$. At this frequency the electric field swings the orientation of water molecules 109 times every second, creating an intense heat that can escalate as quickly as $10{ }^{\circ} \mathrm{C}$ per second (Lew et al., 2002). Water being the predominant component of biological materials, its content directly influences heating. However, there are minor contributions from a host of other factors (Schiffmann, 1986): heating is accelerated by ionic effects (mostly salt content) and specific heat of the composite material (Decareau, 1992). Specific heat is an important property in the 
thermal behaviour of a food subjected to microwaves. Produce with low specific heat may heat very rapidly, and even faster than water of the same weight. Oil heats faster than water due to its much lower specific heat (Schiffmann, 1986). Hence for oily materials, the influence of specific heat becomes the determining factor in microwave heating, owing to the low specific heat of oils, often less than half that of water (Ohlsson, 1983).

\subsection{Microwave interactions with dielectric properties}

When an oscillating electrical field is applied to a polar dielectric, the dipoles within the material attempt to align themselves (polarize) with the field. The rate of change of polarization represents a displacement current in the dielectric and the product of this and the applied field gives the power generated as heat. Averaged over a cycle, the power 'lost' in the material (i.e. dissipated as heat) depends on the phase angle between the applied field and the polarization. For most dielectrics the lag depends on the flexibility of the molecules that house the dipoles, and the randomization effect of temperature.

\section{Flavour generation via Maillard reaction}

The Maillard reaction is incredibly complex. For instance, a simple example such as the reaction of glucose with ammonia gives evidence, using simple methods, of the formation of more than 15 compounds and the reaction of glucose with glycine gives more than 24 . Using HPLC and TLC on solvent-soluble material only $[0.1 \%(w / w)$ of reactants $]$, about 100 components are detectable as reaction products of xylose and glycine (Hodge, 1953).In order to understand something so complex, it is necessary to draw up a simplified scheme of the reactions involved. This has been done most successfully by Hodge in 1953 (Fig. 2).

The discussion here is based on this.

Hodge subdivides the Maillard reaction as follows:

i. Initial stage: products colourless, without absorption in the ultraviolet (about $280 \mathrm{~nm}$ ).

Reaction A: Sugar-amine condensation

Reaction B: Amadori rearrangement

ii. Intermediate stage: products colourless or yellow, with strong absorption in the ultraviolet.

Reaction C: Sugar dehydration

Reaction D: Sugar fragmentation

Reaction E: Amino acid degradation (Strecker degradation)

iii. Final stage: products highly coloured.

Reaction F: Aldol condensation

Reaction G: Aldehyde-amine condensation and formation of heterocyclic nitrogen compounds.

It is worth noting that Mauron (1981) calls the three stages Early, Advanced, and Final Maillard reactions, respectively. The way these reactions fit together is outlined in Fig.2. The 
final products of nonenzymic browning are called melanoidins to distinguish them from the melanins produced by enzymic browning. Theoretically, the distinction is clear; however, in practice, it is very difficult to classify the dark brown products formed in foods, since they tend to be very complex mixtures and are chemically relatively intractable. Reaction $\mathrm{H}$ has been inserted into Fig.2. It represents the much more recently discovered free-radical breakdown of Maillard intermediates. Oxygen plays an essential role in enzymic browning, but is not essential for nonenzymic browning. It may help in fact, for example, in the formation of reductones, such as dehydroascorbic acid, but it may also hinder the progress of the reaction, for example, in oxidising 2-oxopropanal to 2-oxopropanoic acid.

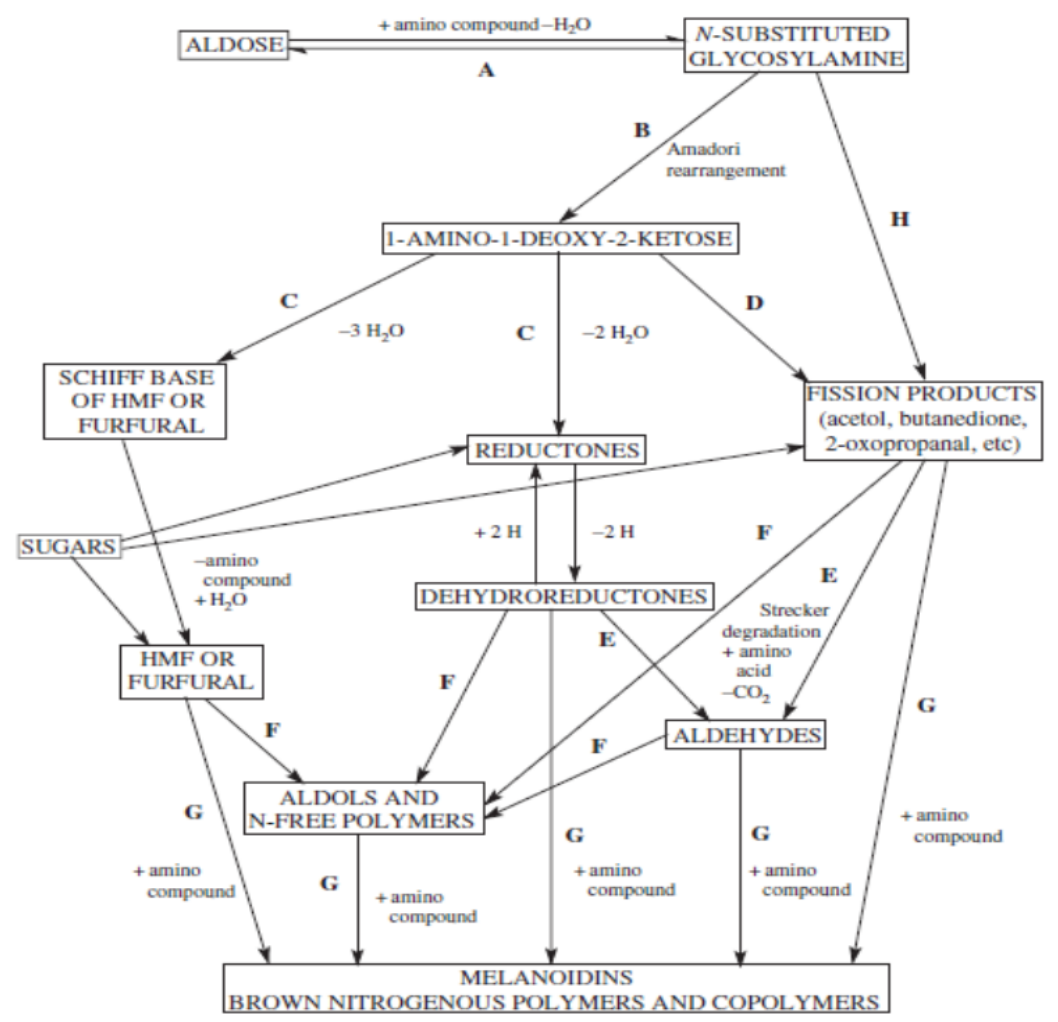

Figure 2. Maillard reaction.

In relation to the flavours produced on exposure to microwave radiation, Yaylayan et al.(1994) examined many combinations of sugars and amino acids, grouping the latter into aliphatic, hydroxylated, aromatic, secondary, basic, amide, acid, and sulfur-containing ones. The odours observed were grouped into eight and they have been assigned to the above groups, as far as possible, below:

Caramel (1); Meaty (4); Nutty (9); Meaty + vegetable Fragrant (6); Baked potato (5) and Baked (3). 
Shibamoto and Yeo (1994) have compared microwave (700 W, high setting, $15 \mathrm{~min}$ ) and thermal treatment (reflux, $100{ }^{\circ} \mathrm{C}, 40 \mathrm{~h}$ ) for a glucose-cysteine system. The conditions used were determined by the onset of browning and aroma formation. The two sets of conditions gave samples with similar popcorn and nutty flavours, but the microwaved samples also gave pungent, raw, and burnt aromas, absent from the conventionally heated ones. The sample prepared conventionally at $\mathrm{pH} 9$ contained much higher amounts of methylpyrazine and 2,6-dimethylpyrazine, whereas the microwaved one gave a much higher amount of 4,5dimethyloxazole and was the only one to produce 2,3-dihydro-3,5-dihydroxy-6-methyl-4Hpyran-4-one. Such data, to some extent, explain the differences in acceptability of the two types of heating. For browning to occur in microwaving, a minimum of $10 \%$ moisture is required. Surprisingly, microwaving at $\mathrm{pH} 2$ gave about twice the absorption at $420 \mathrm{~nm}$ than at $\mathrm{pH} 9$ (about $1 \mathrm{AU})$, the absorption for $\mathrm{pH} 5$ and 7 samples being nearly $0(<0.1 \mathrm{AU})$.

As the Maillard reaction is a series of chemical transformations (Yaylayan, 1997) factors that influence a chemical reaction also affect the Maillard reaction. In general, the rates of chemical reactions depend primarily on temperature, pressure, time, and concentration of reactants. High temperature, pressure, and superheating of reaction solvent associated with microwave irradiation can accelerate simple or single-step chemical reactions such as esterification, hydrolysis and cyclization reactions (Richard et al., 1988; Bose et al., 1994). If the microwave heating is performed under a closed system, then the rate of the microwave reaction accelerates up to 1000 times. However, the time factor plays a crucial role in influencing the product distribution of more complex reactions when carried out under microwave heating. The influence on competitive and consecutive reactions is an important consequence of fast rate of heating under microwave irradiation that is especially pertinent to the propagation of Maillard reaction.

\section{Solvent-mediated Maillard reactions: Model systems}

Given the fact that the Maillard reaction is a complex series of consecutive and competitive reactions, product distribution and intensity of browning will be most affected by microwave irradiation relative to conventional heating. Generally, the final outcome of a Maillard reaction (colour, volatile aroma compounds, and nonvolatile products) depends on temperature, water content, $\mathrm{pH}$, and heating time. Thus, any variation in the reaction parameters will affect the profile of the end products, and hence the perceived aroma and colour. Although simple chemical reactions are fast under microwave irradiation, multistep reactions can remain incomplete or they do not proceed to the same extent as under conventional heating. They produce mixtures that contain the same products (Yaylayan et al., 1994) but with altered distribution patterns. The flavour perception is sensitive to such variations in relative concentrations of different components, especially the character impact compounds, thus drastically changing the sensory properties.

There are few reports in the literature on the microwave-assisted generation of Maillard products using precursors or intermediates. Preparative scale microwave-assisted synthesis (Shui et al., 1990) of Amadori products from D-glucose and amino acids is 
feasible but has not been reported. However, Barbiroli et al. (1978) observed 70-75\% conversion of added glucose/leucine into Amadori compounds with a corresponding decrease in the amount of added amino acid in a bread mix when microwaved for 3 min. Steinke et al. (1989) generated Strecker aldehydes from an aqueous solution of an amino acid and 2,3-butadione (diacetyl) in sealed vials microwaved for $4 \mathrm{~min}$ or heated in a water bath for $60 \mathrm{~min}$ at the same temperature. Significantly higher concentrations of aldehydes were measured in the microwave heated samples. The effect of electrolytes and $\mathrm{pH}$ on the formation of Maillard products during microwave irradiation of aqueous model systems has been studied.

The addition of different salts (Yeo and Shibamoto, 1991a) such as sodium chloride, calcium chloride, and sodium sulfate increased both the intensity of browning and the concentration of flavour compounds. The total volatiles generated from a glucose/cysteine model system (Yeo, and Shibamoto, 1991b) under microwave irradiation has been found to increase with $\mathrm{pH}$. It seems that increasing the $\mathrm{pH}$ and concentration of electrolytes enhances the rate of Maillard reactions under microwave irradiation.

Attempts have been made to compare the chemical composition and yields of volatiles in microwaved and conventionally heated Maillard model systems. However, this type of comparison can be misleading due to the variations in the time-temperature exposure of the two systems under study. In most cases, the temperature of the microwave system is not monitored and time of irradiation is chosen arbitrarily. In order to compare the yields of two systems undergoing the same reaction at different times and temperatures, knowledge of kinetic parameters is required to ascertain whether there are differences in the two processes. Alternatively, the intensity of brown colour formation can be used as an indication that the two systems have undergone equivalent time-temperature exposure. Yaylayan et al. (1994) mimicked actual cooking and surface drying of foods by subjecting the same aqueous sugar/amino acid mixtures to microwave irradiation (640 W) and to conventional heating in an open system, until all the water was evaporated and the residue was dark brown. In order to ensure that both treatments produced the same extent of Maillard reaction for comparison purposes, the conventional heating time was adjusted such that after similar dilutions, both samples had the same spectrophotometric absorption at $460 \mathrm{~nm}$. On the average, $1 \mathrm{~min}$ of microwave heating time produced the same browning extent as $12 \mathrm{~min}$ of conventional heating time. With such treatment, no significant qualitative changes were observed in the composition of both samples, as identified by GC/MS. Parliment (1993) studied, in sealed vials, the products of the Maillard reaction between glucose and proline formed under microwave $(600 \mathrm{~W}$, preheated conventionally for $3 \mathrm{~min}$ and irradiated for $45 \mathrm{~s}$ ) and conventionally heated systems $\left(150^{\circ} \mathrm{C}\right.$ for $\left.15 \mathrm{~min}\right)$. Qualitatively both systems produced similar compounds but in the microwave system Nheterocyclic compounds were present in smaller amounts.

Inhibition of pyrazine formation by natural antioxidants and the foods containing them was measured in a microwaved glucose/glycine model system. Inhibition of lipid oxidation by the same materials was assayed in both bulk and emulsion systems. 
Pyrazines were determined by solid-phase micro extraction followed by GC. Lipid oxidation volatiles were assayed by polyamide fluorescence produced by either a bulk oil display or a hematin- or 2,2'-azobis-(2-amidino-propane) dihydrochloride-accelerated lecithin or fish oil emulsion. It was shown that (i) the inhibition of pyrazine formation depends on high concentrations of water-soluble antioxidants; (ii) such antioxidants occur naturally in some foods and are usually polyphenols; (iii) during pyrazine inhibition, oxidized polyphenols show enhanced nonfluorescing browning similar to enzymic browning products; (iv) monophenols, which structurally cannot form quinone polymers on oxidation, inhibit pyrazines with less browning; (v) during the final pyrazine-forming phase of the Maillard reaction, polyphenolics and reducing agents such as glutathione and ascorbic acid are partially consumed with some nutritional loss; (vi) fruit powders of grape seed, grape skin, and red wine are highly pyrazineinhibitory, steeped blueberry strongly so, but plum purees are moderately pro-pyrazine, and freeze-dried vegetables strongly pro-pyrazine; and (vii) black and green tea infusions are highly inhibitory, whereas spices have mixed effects.

\subsection{Interaction of microwave with the food components}

The major food components - water, carbohydrates, lipids, proteins and salts (minerals) interact differently with MW. Because the primary mechanisms of MW heating are dipole rotation and ion acceleration, MW interactions with foods depend heavily on salt and moisture content. Water selectively absorbs the energy (Mudgett, 1990). In intermediate and high moisture products, the water, not the solids, absorbs the MW energy (Mudgett, 1989; Karel, 1975). However, because of their high heat capacity, they tend to heat unevenly. In drier products, the dissolved salts are concentrated (in the remaining water); if the solids exceed saturation level and precipitate, their ionic conductivities are limited. However, the solids themselves do absorb energy (marshmallow ignition: Mudgett, 1989). Low moisture products generally heat more evenly due to their low heat capacity (Schiffman, 1986).

Alcohols and the hydroxyl groups on sugars and carbohydrates are capable of forming hydrogen bonds and undergo dipolar rotation in an electric field. Low levels of alcohols or sugars in solution in foods have little effect on the interaction of MW with water and dissolved ions. At higher concentrations (jellies, candies), sugars can alter the frequency response of water with MW (Mudgett, 1989).

Proteins have ionizable surface regions that may bind water (or salts), giving rise to various effects associated with free surface charge. Lipids, other than the charged carboxyl groups of the fatty acids, which are usually unavailable due to their participation in the ester linkages of triglycerides, are hydrophobic and interact little with MW if water is present. MW do appear to interact with lipids (and colloidal solids) in low moisture foods as evidenced by energy absorption that cannot be accounted for by either free water or ion activity.

The interaction of microwave energy and food products causes internal heat generation. The rapidly alternating electromagnetic field produces intraparticle collisions in the material, and the translational kinetic energy is converted into heat. For many food products the 
heating is uneven; the outer layers heat most rapidly, depending on the depth of penetration of the energy, and the heat is subsequently conducted into the body of the food. Current research is concerned with achieving uniform heating, especially in relation to pasteurization and sterilization of foods, where non-uniform heating could result in a failure to achieve a safe process. For materials that are electrical conductors $-e . g$. metals, which have a very low resistivity - microwave energy is not absorbed but reflected, and heating does not occur. Short-circuiting may result unless the container is suitably designed and positioned. Metallic containers and trays can effectively improve the uniformity of heating (George 1993; George and Campbell 1994). Currently most packages are made of plastic materials which are transparent to microwave energy. The amount of heat generated in microwave heating depends upon the dielectric properties of the food and the loss factor (see below), which are affected by the food composition, the temperature and the frequency of the microwave energy. For tables of electrical properties of food, and discussion of their application Kent (1987), Ryynanen (1995), and Calay et al. (1995).

The main frequency bands used are 2450 and $896 \mathrm{MHz}$ in Europe and $915 \mathrm{MHz}$ in the USA. Greater penetration and more uniform heating are obtained at the longer wavelengths for food products with low loss factors. Datta and Liu (1992) have compared microwave and conventional heating of foods and concluded that microwave heating is not always the most effective method, especially for nutrient preservation. The effect depends on a variety of properties of the system.

Burfoot et al. (1988) examined the microwave pasteurization of prepared meals using a continuous tunnel device. The product was heated to $80-85^{\circ} \mathrm{C}$ for a few minutes, sufficient to inactivate vegetative pathogenic bacteria, e.g. Salmonella and Campylobacter, but not bacterial spores. The latter are controlled by storing the product below $10^{\circ} \mathrm{C}$. This type of product is not shelf-stable at room temperature and a full sterilization process would be necessary with low acid products of this type to obtain a stable product. Microwave tunnels for this purpose would have to be pressurized to maintain the integrity of the package when sterilizing temperatures $\left(121{ }^{\circ} \mathrm{C}\right)$ had been achieved. A general-purpose plant known as Multitherm has been developed by AlfaStar Ab, Tumba, Sweden (Hallstrom et al. 1988). Burfoot et al. (1996) have modeled the pasteurization of simulated prepared meals in plastic trays with microwaves. Large differences between actual and predicted temperatures were found at some points. For measuring temperatures in microwave systems an invasive fibreoptic probe has been developed, which uses the change in color with temperature of a crystal situated at the end of a glass fibre. Fluoroptic probes are manufactured by Luxtron Corp., CA, USA.

Variations in electric fields, food constituents and the location of the food in a MW oven can lead to nonuniform heating, allowing for less-than-ideal interaction of food components and survival of microorganisms. A number of techniques to improve uniformity of MW heating, such as rotating and oscillating foods, providing an absorbing medium (water) around the product, cycling the power (pulsed power), and varying the frequency and phase, can improve the situation; however, dielectric properties of the food must be known in order to develop effective processes (Yang and Gunasekaran, 2001; Guan et al., 2004). 
Using moisture, salt, and fat content, and temperature $\left(<70^{\circ} \mathrm{C}\right)$ at MW frequencies, Calay et al. (1995) developed polynomial equations to estimate dielectric properties of grains, fruits and vegetables, and meat products. However, they concluded that it was impossible to develop a generic composition based equation. This may be, in part, because as cooking temperature increases, the dielectric constant may increase while the loss factor and depth of penetration decrease (Zheng et al., 1998). The result is that changes in formulation usually require reevaluation with regard to dielectric properties and behavior upon exposure to MW energy.

\subsection{Maillard reaction interaction with food matrix during microwave irradiation}

At the molecular level, the mechanism of heat generation in the microwave oven relies mainly on the interaction of the microwave radiation with dipoles/induced dipoles or with ions. Proteins and lipids do not significantly interact with microwave radiation in the presence of aqueous ions that selectively absorb the radiation. However, in the absence of water, lipids and colloidal solids are known to interact strongly with microwave radiation and the observed levels of energy absorption cannot be explained by the presence of free water and by ion activity (Pomeanz and Meloan, 1987).

Microwave radiation can also interact with alcohols, sugars, and polysachharides. Tightly bound water monolayers do not absorb energy due to hindered molecular rotations. Microwave interactions with a multicomponent system such as food can differ considerably from simple aqueous Maillard model systems, in that "matrix effects" can produce undesirable consequences. Since the core aqueous region of foods are the main sites of interaction with the microwaves, the interior vapor pressure generated as a result can actively force the vapor to the surface of the food, unlike in the conventional oven, where passive migration of water by capillary action to the surface, is diffusion controlled (Schiffmann, 1994a). The water-saturated food surfaces usually remain at relatively cool temperatures of the oven during cooking $\left(40-60^{\circ} \mathrm{C}\right)$, thus preventing browning and crisping (Schiffmann, 1994b). Model studies have already indicated that there are no fundamental differences in the solution phase chemistry of Maillard reaction under microwave irradiation. However, the overall performance of food products under microwave irradiation implies the development of characteristic textural, color and aroma properties similar to that of conventional heating, which differs markedly from microwave heating due to fast rate of heating and "matrix effects."

Food products that rely heavily on Maillard flavours and colours, such as roasted and baked products, perform well in the conventional oven due to the following:

1. The high temperature of the air surrounding the product dehydrates the surface, producing a crust that protects the food from loss of moisture and important aroma volatiles. Dehydration steps are also crucial for the formation of color and aroma precursors by the Maillard reaction.

2. Long time exposure in the conventional oven ensures the completion of slow and/or multistep Maillard reactions responsible for browning and for the generation of specific aromas. 
3. In the case of porous materials such as bread, the high temperature and relative low humidity of the air surrounding the product cause rapid heating of the surface of the food relative to the center, thus creating a temperature and a corresponding inward vapor pressure gradient that helps retain volatile aroma compounds inside the core.

In the microwave oven, the short time exposure and the lack of hot dry air (air being transparent to microwave irradiation) surrounding the surface of the food product not only prevents crusting but also promotes sogginess due to the condensation of the moisture. On the other hand, the rapid release of moisture and its evaporation from the center of the food causes the added and formed volatiles to be "steam distilled" at temperatures below their boiling points. Hence baked and roasted food products, which rely heavily on Maillard produced flavors, usually do not perform well in the microwave oven.

Schiffmann (1994a) summarized the different factors related to microwave ovens that affect aroma generation during cooking of food such as variation in the type of commercial ovens (power, cavity size, .....etc.) and its effect on the reproducibility of performance, speed of heating, oven temperature, and vapor pressure buildup inside the food. The short time required in the microwave oven to attain the same temperature as in the conventional oven not only retards the Maillard reaction but also prevents the establishment of thermal equilibrium throughout the food and uniform temperature distribution through conductive heat transfer. These hot and cold spots in the food product aggravate further the oven hot and cold zones created as a result of standing wave patterns. In addition, different dielectric loss factors $\left(E^{\prime \prime}\right)$ associated with different components in a multi component food product will also contribute to the uneven heating pattern inside the microwave oven. The combined effect of these phenomena is manifested in the excessive exposure of certain parts of food to heat and diminished exposure in others, leading to undesirable textural and flavour modifications such as charring, drying, excessive evaporation, hardening, and development of burnt or raw flavor and aroma notes. The extent of these undesirable modifications is dependent on the size, geometry, thickness, and the composition of the food product. Yeo and Shibamoto (1991c) reviewed the chemical composition of volatiles generated by microwave and conventionally heated food products. White cake batters were cooked to the same degree both in the microwave and the conventional oven. The volatiles released and sensory properties of both products were compared (Whorton and Reineccius, 1989). The number of volatiles detected and the amount of total pyrazines produced were found to be more in the conventionally baked sample. In addition, the microwave cake lacked the nutty, caramel, and browned flavors. In a similar study (Mac-Leod and Coppock, 1976) the number of volatiles generated from boiled beef cooked by microwave for $1 \mathrm{~h}$, was found to be more than the number of volatiles generated by beef boiled conventionally, for the same length of time. When both systems were compared on the basis of "doneness," the microwave sample generated only one third the amount of volatiles detected in the conventional oven. The relative success of the microwave to achieve the Maillard effect of conventional heating may depend to a large degree on the type and composition of the food product. 


\section{Application of microwave technology in some food products}

The use of microwave energy in food processing can be classified into six unit operations: (re)heating, baking and (pre)cooking, tempering, blanching, pasteurisation and sterilisation, and dehydration. Although their objectives differ, these aims are established by similar means: an increase in temperature. Nevertheless, for each special use (different from pure microwave heating), different advantages and disadvantages have to be taken into account. These are presented in the next sections together with some examples of real industrial applications.

Microwaves lend well to speeding up almost any drying processing which the liquid being evaporated is neither explosive nor flammable. The great advantage of microwave drying is speed, often allowing the drying of a material in $10 \%$ or less of the normal drying time. However, in no application, other than laboratory analytical drying systems, are microwaves used to dry a product alone. Always there is the use of additional heat - hot air, ambient or forced circulation; infrared; or some combination of these. In fact, microwave heating, properly applied, and usually represents a minor part of the total heat energy required for drying, the reason being cost.

Also, the following benefits encourage the application of microwave drying technology:

- The products obtain excellent rehydration properties because of the volumetric vaporisation of water, which is a constituent of most food stuffs. When evaporated simultaneously through the whole product piece volume, the water forms capillars inside the product pieces, which produce a high porosity and therefore allow an easy rehydration. In many cases gaining porosity is associated with an expansion of volume, so this process is called "puffing" sometimes. The instant properties are crucial for the application of dried food in the composition of ready meals and additives used in modern citchens.

- The same effect of gaining porosity enables the production of crispy fruit and vegetable snacks.

- In comparison to conventional air belt drying the application of microwaves under vacuum conditions allows fast volumetric drying of the product pieces at relatively low temperatures. So vitamins, taste, flavour and natural colours are conserved very well.

- In comparison to other advanced drying technologies (i.e. freeze drying) microwave vacuum drying is is more economic, as drying progress is much faster and thus allows a higher througput for the same plant dimensions.

- The combination of microwave vacuum drying or puffing and conventional airbelt predrying into a continuous production line is economically most advantageous.

\subsection{Drying}

Drying occurs when water vapor pressure differences between the food interior and exterior drive moisture transfer into the surrounding air. MW drying occurs by both dielectric and conventional heating. When above 50\% moisture, as moisture content 
decreases, dielectric constant and loss factor decrease, especially at higher temperatures. Below 30\% moisture content, MW penetration depth increases sharply (Feng et al., 2002). MW heating in a drying system may adversely affect product quality due to nonuniform temperature distribution and difficulty in controlling product final temperature at low moisture contents.

MW energy can improve quality of fried products. Potato chips can be fried then dried by MW and hot air (Decareau, 1985). MW finish drying to maintain the temperature below the Maillard browning point, of russet burbank potato slices containing $<0.9 \%$ reducing sugar, allows production of chips of acceptable color and texture (Porter, 1971). Potatoes containing $>0.9 \%$ reducing sugar must be removed from the oil at an intermediate moisture content $>13 \%$ to obtain acceptable color of the MW-finished product. Oil content of MW-finished chips may be $90 \%$ that of conventional chips because the fat is absorbed at prefinish moisture levels. Osmotic dehydration prior to MW dehydration efficiently removes water from fruits and preserves volatile flavor compounds. Prothon et al. (2002) osmotically dried apple cubes in $50 \%(\mathrm{w} / \mathrm{w})$ sucrose, then dried them in a MW-assisted drier. Osmotic dehydration reduced drying time required to reach $10 \%$ moisture, but also decreased drying rate and effective moisture diffusivity.

Osmotic pretreatment increased cell wall thickness and increased firmness frehydrated apple pieces, but reduced rehydration capacity. Drying is more efficient when strawberries and blueberries are pretreated with $2 \%$ ethyl oleate and $0.5 \% \mathrm{NaOH}$ (osmotic drying: Venkatachalapathy and Raghavan, 1998, 1999). The osmotic dehydration step was necessary to produce MW-dried strawberries that had similar rehydration ratio, texture, color and sensory properties to freeze-dried berries. Dipping blueberries in $2.5 \%$ ethyl oleate and $0.2 \%$ $\mathrm{NaOH}$ followed by sucrose osmotic dehydration prior to $\mathrm{MW}$ drying treatment reduces drying (from $>80 \%$ to $15 \%$ moisture) time to one-twentieth of that needed for tray drying (Feng et al., 1999). MW-dried frozen berries had a higher rehydration ratio. MW drying generated three unique flavour compounds (2-butanone, 2-methyl butanal, and 3-methyl butanal) while freeze-dried berries lost several, including the typical blueberry aroma, 1,8cineole. Compared with hot-air dried berries, MW-dried cranberries have better color, softer texture and similar storage stability at room temperature (Yongsawatdigul and Gunasekaran, 1996). Vacuum permits water vaporization at a lower temperature and at a faster rate than at atmospheric pressure. Application of vacuum reduces the boiling point of water and the drying temperatures. Combining vacuum and MW drying (VMD) reduces or avoids the heat and rate limitations at atmospheric pressure (Durance and Wang, 2002). MW energy is an efficient mechanism of energy transfer through the vacuum and into the interior of the food. Drying time for carrots has been shown to be $30 \%$ less for a combination of VMD and hot air drying than that of a conventional hot air drying method (Baysal et al., 2002). No constant rate period existed and drying occurred mainly during the falling rate period. No differences occurred in dry matter content, bulk density or porosity; however, aw and color ( $\mathrm{L}, \mathrm{a}, \mathrm{b}$ values) were higher and rehydration capacities were higher in carrots dried by the combination method. Fruit and vegetable variety can have significant effects on the VMD process. 
After blanching potatoes prior to VMD to produce fat-free chips, Lefort et al. (2003) reported that yellow flesh cultivars had lower moisture content and higher specific gravity, starch content, and crispness scores than red flesh cultivars. The authors concluded that cultivars low in specific gravity and starch content produced chips with a crispy but less rigid texture, which are desirable characteristics for chips produced by VMD. Color was unaffected. A $\mathrm{CaCl} 2$ pretreatment prior to $\mathrm{MW}$-assisted $\mathrm{AD}$ increases the hardness of rehydrated apples and potatoes (Arhne et al., 2003). Water loss rates are similar during drying at $50{ }^{\circ} \mathrm{C}$, but at $70{ }^{\circ} \mathrm{C}$ rates in potatoes are slower Retention of volatiles makes VMD an attractive preservation method for herbs and spices. Parsley subjected to VMD is greener immediately and after 8 weeks than hot air-dried samples (Boehm et al., 2002). VMD preserved more than $90 \%$ of the essential oils compared to $30 \%$ by hot air drying and resulting in higher parsley-like and green-grassy aroma and less hay/straw-like off-flavor. MW drying of a variety of herbs, requiring 10 to $16 \mathrm{~min}$, affected color, appearance, aroma and relative reconstitution capacity (RRC; Fathima et al., 2001). The RRC for dried coriander, mint, fenugreek, shepu and amaranthus was 10.3, 10.3, 31.7, 32.8, and 38.3 respectively. Herbs with the lowest RRC (mint, coriander), had the lowest scores for flavor and color scores, while dried amaranthus, with the highest RRC, had scores similar to that of the fresh herb. Storage $(60 \mathrm{~d})$ results in little change in sensory properties. Working with garlic, Sharma and Prasad (2001) reported that in comparison with hot air drying $\left(70{ }^{\circ} \mathrm{C}\right)$ alone, VMD reduced drying time by $80-90 \%$ and dried garlic products had higher sensory quality scores. Yousif et al. (1999) found that VMD basil yielded 2.5 times the linalool and 1.5 times methylchavicol (the major volatiles) as air-dried samples. VMD basil had more volatiles than fresh basil due to chemical reactions during drying. AD basil was darker and less green. VMD samples had a higher rehydration rate, while the potential of the plant material to rehydrate was hindered in $\mathrm{AD}$ samples possibly due to maintenance of structural integrity of the cells.

Begum and Brewer (2001) studied the physical, chemical and sensory quality of snow peas blanched by boiling water, steam, microwave and microwave blanching in heat-sealable bags. No differences occurred in lightness $L$ values. Boiling water-blanched peas were the least green, with low a values, whereas steam and microwave blanched in bag peas were the most green. Boiling water-blanched peas had the least $b$ value whereas there was no significant difference in the $b$ values for all other blanching treatments. Ascorbic acid, one of the most labile nutrients in vegetable is water soluble and sensitive to $\mathrm{pH}$, light and heat and is affected by the naturally occurring enzyme ascorbic acid oxidase. Preservation of ascorbic acid in vegetables, particularly those that are good sources, is important in preserving food quality (Brewer and Begum, 2003). Ascorbic acid losses in fruits and vegetables are inevitable and all blanching treatments result in some reduced ascorbic acid losses. Boiling water blanching produced the lowest reduced ascorbic acid content in snow peas while all other blanching treatments resulted in $31-32 \mathrm{mg} / 100 \mathrm{~g}$. At higher power and longer times actual reduced ascorbic acid content increased, but when adjusted for moisture losses, reduced ascorbic acid content decreased. Lane et al. (1985) studied the ascorbic acid content of four vegetables blanched by microwave and conventional methods (boiling water and 
steaming). Their results suggested that with the exception of steam-blanched purple hull peas, ascorbic acid retention was not affected by the blanching method. Drake et al. (1981) studied the influence of blanching method on the quality of selected vegetables. Water-and steam-blanched asparagus and green beans had similar ascorbic acid concentrations and both were superior in ascorbic acid to the microwave-blanched product. Microwave and steam-blanched green peas contained less ascorbic acid than water-blanched green peas.

\subsection{Baking}

Microwave baking has been the focus of much research and development since the 1950s, with variable success (Seyhun et al., 2003). All results point to the general rule that to achieve success, considerable product reformulation must be considered (Decareau, 1992). Baking with various emulsifiers, gums, starches, fat contents and enzymes has been widely investigated (Ozmutlu et al., 2001; Sumnu, 2001; Keskin et al., 2004). With adequate product formulation, microwave baking can offer good quality products with high convenience. Pillsbury ${ }^{\circledR}$ has a new line of frozen biscuits and dinner rolls specifically designed for the microwave to deliver warm, soft, ready-to-eat bread rolls in $25 \mathrm{~s}$.

The browning reactions in baked products are the result of heating of reducing sugars with proteins or nitrogen-containing substances to form compounds like melanoidins, and start at around $160{ }^{\circ} \mathrm{C}$ (Matz, 1960). When sugars such as fructose, maltose and dextrose are heated to around $171{ }^{\circ} \mathrm{C}$, molecules are combined to form coloured substances called caramels. A relatively low food surface and low surrounding temperatures in microwave baking do not enable the browning reactions to occur. Moreover, in microwave ovens, evaporated water molecules from the food system directly interact with cold air around the product and condense, which prevents browning and crisping reactions (Schiffmann, 1994). Dough products which are expected to be crisp and brown become soggy after baking. When heated for a longer period, they become dry and brittle but never brown. Brown surfaces achieved by Maillard reactions and caramelization of sugars are a result of high temperature accompanied by dehydration (Burea et al., 1987). In addition, time is necessary for completion of these browning reactions. The kinetic rate constant of browning reaction increases with increased temperature (Ibarz et al., 2000) and decreased moisture content (Moyano et al., 2002).

Good browning and flavour development were attained when no water was added to the system. The characteristic baked/roasted aroma produced decreased as moisture content increased. Flavour development was still apparent in the 5\% moisture system (GC/MS analysis of flavour compounds) despite very little browning. Electrolytes $(0-0.5 \mathrm{M} \mathrm{NaCl}$, $\mathrm{CaCl}_{2}, \mathrm{FeCl}_{2}$, or $\mathrm{NaSO}_{3}$ ) enhanced both flavor production and browning intensity in an Lcysteine/D-glucose model system. $\mathrm{NaCl}$ promoted the development of the greatest amount of volatiles (seven times the control) and $\mathrm{FeCl}_{2}$ the least (three Microwave processing, nutritional and sensory quality 93 times the control). $\mathrm{NaCl}$ produced the most browning while $\mathrm{FeCl}_{2}$ produced the least. Browning treatments for breads were evaluated. When susceptors were used with MW baking, desired browning and hardness were obtained on 
the bottom surfaces of the breads but did not affect surface color significantly. Breads coated with the solution containing sodium bicarbonate $(10.5 \%)$, glucose $(31.6 \%)$ and glycine $(5.3 \%)$ did not have the desired crust colour or hardness, while conventional browning at $200{ }^{\circ} \mathrm{C}$ achieved browning on top and bottom surfaces and crust formation on the bottom surface in $8 \mathrm{~min}$.

MW-baked dough products are often of lower quality than conventionally baked products. Differences in heat and mass transfer patterns, insufficient starch gelatinization due to very short MW baking times, MW-induced changes in gluten, and rapid generation of gas and steam result in crustless products which are tougher and coarser and have less firm textures than conventionally baked products. They often have reduced height, gummy texture, hard crumb, and an undesirable moisture gradient along the vertical axis of the product. During baking, two simultaneous processes occur: (1) energy (heat) is transferred to the food and (2) this causes changes (starch gelatinization, protein denaturation) within and at the surface of the product. In conventional baking, the pattern of temperature rise in the interior differs substantially from that near its surface. During MW heating, the dough near the surface is heated instantaneously but heat must be transferred to the interior via conduction. This instantaneous surface heating promotes nearly instantaneous water evaporation as well. In addition, the cellular structure of dough makes it a poor heat conductor.

Flavours generated as a result of browning reactions are also absent in microwave baked products. The aroma profile of a microwave baked cake was shown to be similar to that of batter. Many of the nutty, brown and caramel-type aromas observed in the conventional cake were lacking in microwave baked cakes (Whorton and Reineccius, 1990). Individual flavour components are subjected to losses through distillation, flavour binding by starches and proteins and chemical degradation during microwave baking. Crust also provides a barrier against the loss of flavours (Eliasson and Larsson, 1993). Flavours can easily be released from microwave baked product due to the absence of crust.

Changing the food formulation to reduce compound volatility minimizes loss of flavour compounds during microwave baking. This can be done by adding an oil phase or increasing the oil content (Yaylayan and Roberts, 2001). Flavouring agents may be encapsulated to reduce the volatility of aroma compounds (Whorton and Reineccius, 1990). Unwanted flavours such as flour or egg-like flavours develop during microwave baking of cakes. Flavouring agents may be added to mask these unwanted flavours and obtain a similar flavour profile to conventionally baked cakes. Since products baked in microwave ovens have inferior quality, improving this quality represents a challenge to food technologists. Therefore, a thorough understanding of the effects of microwaves on the major ingredients in baked products such as starch and gluten will play an important role in improving the quality of these products.

\subsection{Cooking}

Microwave heating of food products is done in a relatively quick time period as compared to conventional oven cooking. The flavour of the final product can result from aroma 
generated during microwave cooking. It can also be already contained in the food, e.g., in a precooked meal. In any case, the release phenomenon is the same and it is the timing that may be different. If the flavour is only produced at the end of heating, losses due to volatilization will be diminished considerably. In microwave heating of a precooked product, the volatile aroma compounds are integral to its final aroma, and losses can imbalance the aroma. Aroma is defined as the volatile aroma compounds that contribute both to the orthonasal (sniffing) and retronasal (eating) smell of a food. This section will explain the theory and give examples of how aroma compounds present in microwave foods can be lost during cooking.

The impact of microwave cooking on the formation of early Maillard products was investigated and compared with the effect of conventional cooking, using milk as a test system. Experiments were carried out at controlled temperatures of $80^{\circ} \mathrm{C}$ and $90^{\circ} \mathrm{C}$, respectively, at holding times up to $420 \mathrm{~min}$. Hydroxymethylfurfural (HMF) and lactulose, which are all established indicators to estimate heat damage, were determined. The concentrations of all the heating indicators increased with increasing heating time. For example in the $90^{\circ} \mathrm{C}$ test series the furosine values rose from $34 \mathrm{mg} \mathrm{litre}^{-1}(0.5 \mathrm{~h})$ to $94 \mathrm{mg}$ litre $^{-1}\left(2 \mathrm{~h}\right.$ holding time) in the milk heated by microwaves and from $35 \mathrm{mg}^{-1 i t r e} \mathrm{e}^{-1}(0.5 \mathrm{~h})$ to 96 mg litre ${ }^{-1}(2 \mathrm{~h})$ in the conventionally heated milk. None of the reaction products showed significant differences as between the microwave heating and conventional cooking methods (Katz, 1994).

Flavour may be a problem in MW-cooked foods because flavour volatiles distill off, bind to proteins and other molecules or fail to develop at all. A number of methods have been developed to prevent or offset these flavor problems. Extraction process wherein substrates are mixed with MW-transparent solvent and exposed to MW which liberates target compounds from natural materials (e.g. spices). Selectivity can be varied by altering solvents/conditions. MW extraction in combination with liquid $\mathrm{CO}_{2}$ can be used as an alternative to supercritical fluid extraction (decaffeination of coffee, defatting of cocoa powder). A process to generate desirable aromas when a food and/or package is subjected to MW radiation. The aroma-generating material, consisting of a sugar alone or in combination with an amino acid source, and an effective amount of a MW susceptible material for conductive heat transfer sufficient to catalyze the desired chemical reactions

Vegetables are often cooked to increase palatability and digestibility, ascorbic acid content of MW-cooked, frozen peas was lower, retention of chlorophyll and organic acids (lactic, succinic, malic, citric.....etc) was higher for peas cooked without water. Effects were smaller for carrots. Those cooked without water had higher flavor scores and carotene retention than those cooked with water. While MW cooking of vegetables generally results in better nutrient retention, there is no one method that produces overall superior sensory characteristics when considering color, flavor, texture, and moistness.

Cooking starchy tubers gelatinizes the starch softening the texture. After a lag of $4 \mathrm{~min}$, water loss during MW cooking of potatoes was rapid and linear. Starch gelatinization began at the surface and in the center, and then spread throughout the tuber cross-section after 1 
min. Results suggest that the MW cooking process is divided into two phases: (1) the MW energy input raises the internal temperature to about $100{ }^{\circ} \mathrm{C}$, then $(2)$ water is vaporized at a constant temperature. Immersing potatoes in boiling water after the first phase prolonged cooking time compared to MW heating, suggesting that MW treatment affects texture by a mechanism independent of the thermal profile induced by cooking.

\section{Conclusion}

This chapter reviewed the flavours and colors appropriate for microwave foods. It discusses the types of flavor definition, the sources of natural flavours and the difference between conventional and microweave heating as well as the generation of flavour from microwave heating especially via maillard reaction. It isolates the particular effects of microwave heating on the browning reaction in flavor formation and its implications for the choice and application of flavours for microwave foods. For products that are simply seasoned and reheated, the microwave does not present significant challenges that are different from conventional heating. Microwave popcorn is an ongoing challenge to create a good flavour that will not all be volatilized during heating. Encapsulation can provide a benefit in protecting the flavour during processing and helping to retain it during the popping process. As work continues to better understand flavours, there will be new developments that will benefit microwave food products.

\section{Author details}

G.E. Ibrahim, A.H. El-Ghorab*, K.F. El-Massry and F. Osman

Chemistry of Flavour and Aroma Department, National Research Center, Tahrir St. Dokki, Cairo, Giza, Egypt

\section{References}

Arhne, L., Prothon, F., and F., T. (2003). Comparison of drying kinetics and texture effects of two calcium pretreatments before MW-assisted dehydration of apple and potato', Internat J Food Sci and Technol, 38(4), 411-420.

Barbiroli, G.; Garutti, A. M. and Mazzaracchio, P.( (1978). Note on behavior of 1-amino-1deoxy-2-ketose derivatives during cooking when added to starch based foodstuffs. Cereal Chem. 55:1056-1959.

Baysal, T., Ersus, S., and Icier, F. (2002). Effects of microwave and hot air combination drying on the quality of carrot', Food Sci and BioTechnol, 11(1), 19-23.

Begum, S. and Brewer, M. S. (2001). Physical, chemical and sensory quality of microwave blanched snow peas. Journal of Food Quality, 24(1), 479-493.

Boehm, M., Bade, M., and Kunz, B. (2002). Quality stabilisation of fresh herbs using a combined vacuum-microwave drying process. Adv in Food Sci, 24(2), 55-61.

${ }^{*}$ Corresponding Author 
Bose, A. K.; Manhas, M. S.; Banik, B. K. and Robb, E. W. (1994). Microwave-induced organic reaction enhancement. (MORE) chemistry: Techniques for rapid, safe and inexpensive synthesis. Res. Chem. Interned. 20:1-11.

Brewer, M. S. and Begum, S. (2003). Effect of microwave power level and time on ascorbic acid content, peroxidase activity and colour of selected vegetables. Journal of Food Processing and Preservation, 27, 411-426.

Burea, M. P., Chiriife, J., Resnik, S. L. and Lozano, D. R. (1987). Nonenzymatic browning in liquid model systems of high water activity: Kinetics of colour changes due to caramelization of various sugars. J Food Sci, 52(4), 1059-1062.

Burfoot, D., Griffin, W. J. and James, S. J. (1988). Microwave pasteurization of prepared meals. J. Food Eng., 8(3), 145-156.

Burfoot, D., Railton, C. J., Foster, A. M. and Reavell, S. R. (1996). Modelling the pasteurization of prepared meals with microwaves at $896 \mathrm{MHz}$. J. Food Eng., 30(1/2), 117-133.

Calay, R.K., Newborough, M., Probert, D., and Calay, P.S. (1995). Predicative equations for the dielectric properties of foods. Int J Food Sci Technol, 29, 699-713.

Datta, A. K. and Liu, J. (1992). Thermal time distributions for microwave and conventional heating of food. Food \& Bioproducts Processing, Trans. IChemE, 70, C2, 83-90.

Decareau, R. V. (1992). Microwave Foods: New Product Development, Food and Nutrition Press, Inc., Connecticut.

Decareau, R.V. (1985). Microwaves in the Food Processing Industry, Academic Press, New York.

Drake, S. R.; Spayd, S. E. and Thompson, J. B. (1981). The influence of blanch and freezing methods on the quality of selected vegetables. Journal of Food Quality, 4(4), 271-278.

Eliasson, A. C. and Larsson, K. (1993). Cereals in Breadmaking: A Molecular Colloidal Approach, Marcel Dekker, New York.

Fathima, A., Begum, K., and Rajalakshmi, D. (2001). Microwave drying of selected greens and their sensory characteristics. Plant Foods for Human Nutr, 56(4), 303-311.

Feng, H., Juming, T., and Cavalieri, R.P. (2002). Dielectric properties of dehydrated apples as affected by moisture and temperature. Transactions of the ASAE, 45(1), 129-135.

Feng, H., Juming, T., Mattinson, D.S. and Fellman, J.K. (1999). Microwave and spouted bed drying of frozen blueberries: The effect of drying and pretreatment methods on physical properties and retention of flavor volatiles. J. Food Proc and Preserv, 23(6), 463-479.

George, R. M. (1993). Making (micro)waves. Food Processing, 62(5), 23-28.

George, R. M. and Campbell, G. M. (1994). The use of metallic packaging to improve heating uniformity and process validation during microwave sterilization. In R. Field (Ed.), Food Process Engineering, IChemE Symposium, (pp. 219-225), Univ. Bath, UK.

Giese, J. (992). dvances in microwave food processing. Food Technology Special Report, 118-123.

Giguere, R. J.; Bray, T. L.; Duncan, S. M. and Majetich, G.(1986). Application of commercial microwave ovens to organic synthesis. Tetrahedron Lett. 27:4945-4948. 
Guan, D., Cheng, M., Wang, Y., and Tang, J. (2004). Dielectric properties of mashed potatoes relevant to microwave and radio-frequency pasteurization and sterilization processes. J Food Sci, 69(9), 30-37.

Hallstrom, B., Skjoldebrand, C. and Tragardh, C. (1988). HeattTransfer and food products, London: Elsevier Applied Science.

Hodge, J. E. (1953). Chemistry of browning reactions in model systems, J. Agric. Food Chem., 1, 928-943.

Ibarz, A., Pagan, J. and Garza, S. (2000). Kinetic models of non-enzymatic browning in apple puree. J Sci Food Agric, 80(8), 1162-1168.

Karel, M. (1975). Physiochemical modification of the state of water in foods', in Duckworth, RB, Water Relations in Foods, Academic Press, New York.

Katz, I. (1994). Maillard, microwave and extrusion cooking: generation of aromas, in 'Thermally Generated Flavours: Maillard, Microwave and Extrusion Processes', T.H. Parliment, M.J. Morello and R. J.McGorrin (eds), American Chemical Society,Washington DC, 2-6.

Kent, M. (1987) Electric and dielectric properties of food materials. London: Science and Technology Publishers.

Keskin, S. O., Sumnu, G. and Sahin, S. (2004). Usage of enzymes in a novel baking process. Nahrung/Food, 48(2): 156-160.

Lane, R. H.; Boschung, M. D. and Abdel-Ghany, M. (1985). Ascorbic acid retention of selected vegetables blanched by microwave and conventional methods. Journal of Food Quality, 8(2\&3), 139-144.

Lefort, J.F., Durance, T.D. and Upadhyaya, M.K. (2003). Effects of tuber storage and cultivar on the quality of vacuum microwave-dried potato chips. J Food Sci, 68(2), 690-696.

Lew, A., Krutzik, P. O., Hart, M. E. and Chamberlin, A. R. (2002). Increasing rates of reaction: microwave-assisted organic synthesis for combinatorial chemistry, Journal of Combinatorial Chemistry, 4(2): 95-105.

Mac-Leod, G. and Coppock, B. M. (1976). Volatile flavor components of beef boiled conventionally and by microwave radiation. J. Agric. Food Chem. 24:835-843.

Matz, S. A. (1960). Bakery Technology and Engineering, AVI Publishing, Westport, CT.

Mauron, J. (1981). The Maillard reaction in food; a critical review from the nutritional viewpoint. A, 5-35.

Moyano, P. C., Rioseco, V. K. and Gonzalez, P. A. (2002). Kinetics of crust colour changes during deep-fat frying of impregnated french fries. J Food Eng, 54: 249-255.

Mudgett, R.E. (1989). Microwave food processing: scientific status summary. Food Technol, 42(1), 117-126.

Mudgett, R.E. (1990). Developments in microwave food processing', in Schwartzberg, HG and Rao, MA, BioTechnology and Food Process Engineering, Marcel Dekker, New York, pp. 359-404.

Ohlsson, T. (1983). Fundamentals of microwave cooking, Microwave World, 4(2): 4. Code of Federal Regulations (2008). Title 21, Vol. 2, US Government Printing Office, Washington, DC.

Ozmutlu, O., Sumnu, G. and Sahin, S. (2001). Effects of different formulations on the quality of microwave baked breads. European Food Research and Technology, 213(1):38-42. 
Paré, J. R.; Sigouin, M. and Lapointe, J. (1991). U.S. Pat. No 5,002,784 issued March 26.

Parliment, T. H. (1993). Comparison of thermal and microwave mediated Maillard reactions, in 'Food Flavours, Ingredients and Composition', G. Charalambous (ed), Elsevier Science Publishers, Amsterdam, 657-662.

Peterson, E. R. (1993). Microwave chemistry: A conceptual review of the literature. In Quality Enhancement Using Microwaves. 28th Annual Microwave Symposium Proceedings, International Microwave Power Institute.

Pillsbury, a division of General Mills, http://www.generalmills.com

Pomeanz, Y. and Meloan, C. E. (1987). Food Analysis: Theory and Practice, $2^{\text {nd }}$ ed. Van Reinhold Nostrand, New York.

Porter,V.L. (1971). The effect of microwave finish-drying and other process factors on quality of potato chips', PhD Thesis, Univ. of Illinois, Dissertation Abs. Internat., Sect. B, Sci. and Eng., 31(9), 5414.

Prothon, F., AHRNE, L.M., Funebo, T., Kidman, S., Langton, M., and Sjoholm, I. (2002). Effects of combined osmotic and microwave dehydration of apple on texture, microstructure and rehydration characteristics. Lebensmittel-Wissenschaft und -Technol, 34(2), 95-101.

Richard, N., Gedye, F. E. and Kenneth, C. W. (1988). The rapid synthesis of organic compounds in microwave ovens. Can. J. Chem. 66:17-26.

Ryynanen, S. (1995). The electromagnetic properties of food materials. J. Food Eng., 26(4), 409-429.

Schiffman, R.F. (1986). Food product development for microwave processing. Food Technol, 40(6), 94-96.

Schiffmann, R. F. (1986). Food product development for microwave processing, Food Technology, June 1986: 94-98.

Schiffmann, R. F. (1994a). Critical factors in microwave generated aromas', in McGorrin J, Parlimant T H and Morello M J, Thermally Generated Flavors: Maillard,Microwave and Extrusion Processes, ACS Symposium Series No. 543, American Chemical Society, Washington DC, 386-394.

Schiffmann, R. F. (1994b). Critical factors in Microwave-generated aromas. In Thermally Generated Flavors: Maillard, Microwave and Extrusion Processes (J. McGorrin, T. H. Parlimant, and M. J. Morello, eds.). ACS Symposium Series, No. 543, American Chemical Society, Washington DC, pp. 386-394

Seyhun, N., Sumnu, G. and Sahin, S. (2003). Effects of different emulsifier types, fat contents, and gum types on retardation of staling of microwave-baked cakes, Nahrung/Food, 47(4): 248-251.

Sharma, G.P. and Prasad, S. (2001). Drying of garlic (Allium sativum) cloves by microwavehot air combination. J Food Eng, 50(2), 99-105.

Shibamoto, T. and Yeo, H. (1994). Flavor in the cysteine-glucose model system prepared in microwave and conventional ovens, in Thermally Generated Flavors: Maillard, Microwave, and Extrusion Processes, T. H. Parliment, M. J. Morello, and R. J. Mc-Gorrin (eds), American Chemical Society, Washington, DC, 457-465. 
Shui, T. C., Shyh, H. C. and Kung, T. W. (1990). Preparative scale organic synthesis using a kitchen microwave oven. J. Chem. Soc., Chem. Commun.11:807-809.

Steinke, J. A.; Frick, C. M. ; Gallagher, J. A. and Strassburger. K. J.(1989). Influence of Microwave heating on flavor. In Thermal Generation of Aromas (T. Parliment, R. McGorrin, and C. T. Ho, eds.). ACS Symposium Series, No. 409, American Chemical Society, Washington, DC, pp. 520-525.

Sumnu, G. (2001). A review on microwave baking of foods, International Journal of Food Science and Technology, 36: 117-127.

van Eijk, T. (1994). In Thermally Generated Flavours: Maillard, Microwave and Extrusion Processes, ed. by Parliment, T.H., Morello, M.J. and McGorrin, R.J., American Chemical Society, Washington, DC, pp. 395-404.

Venkatachalapathy, K. and Raghavan, G.S.V. (1998). Microwave drying of osmotically dehydrated blueberries', J Microwave Power and Electromag Energy, 33(2), 95-102.

Venkatachalapathy, K. and Raghavan, G.S.V. (1999). Combined osmotic and microwave drying of strawberries. Drying Technol, 17(4/5), 837-853.

Whorton, C. and Reineccius, G. (1989). Flavour development in a microwaved versus a conventionally baked cake. In Thermal Generation of Aromas (T. Parliment, R. McGorrin, and C. T. Ho, eds.). ACS Symposium Series, No. 409; American Chemical Society, Washington, DC, p. 526.

Whorton, C. and Reineccius, G. (1990). Current developments in microwave flavors', Cereal Foods World, 35(6), 553-559.

Yang, H.W. and Gunasekaran, S (2001). Temperature profiles in a cylindrical model food during pulsed microwave heating. J Food Sci, 66(7), 998-1004.

Yaylayan, V. (1997). Classification of the Maillard reaction: A conceptual approach. Trends Food Sci. Technol. 8:13-18.

Yaylayan, V. A. and Roberts, D. D. (2001). Generation and release of food aromas under microwave heating', in Datta A K and Anantheswaran R C, Handbook of Microwave Technology for Food Applications, Marcel Dekker, New York, 173-189.

Yaylayan, V.; Eorage, N. G. and Mandeville, S.(1994). Microwave and thermally induced Maillard reactions. In Thermally Generated Flavors: Maillard, Microwave and Extrusion Processes. J. McGorrin, T. H. Parlimant, and M. J. Morello (eds.). ACS Symposium Series, No. 543, American Chemical Society, Washington DC, pp. 449-456.

Yeo, H. C. H. and Shibamoto, T. J. (1991a). Microwave-induced volatiles of the Maillard model system under different $\mathrm{pH}$ conditions. J. Agric. Food Chem. 39:370-373.

Yeo, H. C. H. and Shibamoto, T. J. (1991b) Flavour and browning enhancement by electrolytes during microwave irradiation of the Maillard model systems. J. Agric. Food Chem. 39:948-951.

Yeo, H.C.H. and Shibamoto, T. (1991c). Chemical comparison of flavours in microwaved and conventionally heated foods. Trend FoodSci. Technol., 2, 329-332.

Yongsawatdigul, J. and Gunasekaran, S. (1996). Microwave-vacuum drying of cranberries. Part I. Energy use and efficiency', 'Microwave-vacuum drying of cranberries. Part II. Quality evaluation. J Food Proc and Preserv, 20(2), 121-143. 
Yousif, A.N., Scaman, C.H., Durance, T.D., and Benoit, G. (1999). Flavour volatiles and physical properties of vacuum-microwave and air-dried sweet basil (Ocimum basilicum L). J Agric Food Chem, 47, 4777-4781.

Zheng, M., Huang, Y.W., Nelson, S.O., Bartley, P.G. and Gates, K.W. (1998). Dielectric properties and thermal conductivity of marinated shrimp and channel catfish. J. Food Sci, 63(4), 668-672. 